\title{
Construcción de un instrumento para evaluar el razonamiento prosocial en niños de 7 y 8 años: una versión pictórica*
}

\author{
Construction of an Instrument Designed to Assess Prosocial \\ Reasoning in Children Aged 7 to 8: a Graphic Version
}

Recibido: octubre 2 de 2009 | Revisado: noviembre 14 de 2009 | Aceptado: enero 11 de 2010

\author{
Viviana Noemí Lemos ** \\ Centro Interdisciplinario de Investigaciones en Psicología \\ Matemática y Experimental, Buenos Aires, Argentina \\ Universidad Adventista del Plata, Entre Ríos, Argentina \\ María CRistina Richaud de Minzi ${ }^{* * *}$ \\ Centro Interdisciplinario de Investigaciones en Psicología \\ Matemática y Experimental, Buenos Aires, Argentina
}

Para citar este artículo. Lemos, V.N. \& Richaud de Minzi, M.C. (2010). Construcción de un instrumento para evaluar el razonamiento prosocial en niños de 7 y 8 años: una versión pictórica. Universitas Psychologica, 9 (3), 879-891.

* Artículo de investigación en psicometría. Proyecto de investigación subsidiado por la Facultad de Humanidades, Educación y Ciencias Sociales de la Universidad Adventista del Plata (UAP) y el Consejo Nacional de Investigaciones Científicas y Técnicas (CONICET).

** Centro Interdisciplinario de Investigaciones en Psicología Matemática y Experimental (CIIPME) dependiente del Consejo Nacional de Investigaciones Científicas y Técnicas (CONICET). Centro de Investigaciones en Psicología y Ciencias Afines (CIPCA) de la Universidad Adventista del Plata. 25 de Mayo 99, 3103 Libertador San Martín, Entre Ríos. Argentina. Correos electrónico: vivianalemos@doc.uap.edu.ar

**** Centro Interdisciplinario de Investigaciones en Psicología Matemática y Experimental. Tte. Gral. J.D. Perón 2158, Cap. Fed., Buenos Aires, Argentina. Correos electrónico: minzi@ciudad.com.ar

RES UMEN

El objetivo de este estudio fue desarrollar un instrumento que permita evaluar el razonamiento prosocial en niños de 7 y 8 años. En este trabajo, se presentan los procedimientos seguidos y resultados psicométricos de una versión con apoyo pictográfico.

Se observó una consistencia en la jerarquía de los razonamientos prosociales empleados por los niños, en cada una de las historias que componen el instrumento. Los índices de consistencia interna obtenidos fueron aceptables, en función de la edad de los niños y de la complejidad del constructo evaluado. Se obtuvieron también, resultados consistentes con la teoría, en cuanto a la relación entre los tipos de razonamiento prosocial, y a las diferencias encontradas por sexo y edad, que apoyan la validez constructiva del instrumento. Palabras clave autores

Evaluación infantil, habilidades sociales, conducta prosocial, razonamiento prosocial.

Palabras clave descriptor

Niños (7-8 años), aspectos psicológicos, conducta infantil, conducta social, pruebas psicológicas.

\section{A B S T R ACT}

The aim of this study was to develop an instrument that would allow the assessment of prosocial thinking in children of 7 and 8 years of age. This work presents the procedures that were followed and the psychometric results of a version with pictographic support.

A consistency was observed in the hierarchy of prosocial reasoning applied by children in each of the stories used in this instrument. The indexes of internal consistency were acceptable, based on the age of the children and the complexity of the construct being assessed. Results consistent with the theory were also found, as regards the relationship between the types of prosocial reasoning and the differences between sex and age, which support the construct validity of the instrument.

Key words authors

Child Assessment, Social Abilities, Prosocial Conduct, Prosocial Reasoning. Key words plus

Children (7-8 years), Psychological Aspects, Child Behavior, Social Behavior, Psychological Tests. 


\section{Introducción}

La interacción social es la base del desarrollo humano, por lo que es de máxima importancia establecer relaciones interpersonales adecuadas. El conocimiento social se construye con base en los sistemas conceptuales moral, convencional y personal, que son las categorías fundamentales de la experiencia social (Turiel, 1983). El sistema moral se desarrolla a partir de las experiencias de determinados sucesos con implicaciones objetivas o intrínsecas para la justicia, los derechos y el daño/ bienestar de los demás; el convencional, a partir de los juicios sobre lo bueno y lo malo y de la normatividad de las convenciones, que no derivan de su naturaleza intrínseca sino de las demandas extrínsecas impuestas por el consenso social, arbitrarias y alterables, y que facilitan las interacciones sociales. Por último, el sistema personal deriva de la libre elección de la persona, en función de sus gustos y preferencias.

Según Pérez-Delgado (1995), "El juicio moral es uno de los componentes más importantes del desarrollo moral y uno de los determinantes más decisivos de la conducta moral. Esencialmente, el juicio moral o razonamiento moral se refiere a los procesos para decidir si el curso de la acción dentro de un dilema específico es mejor moralmente, es decir, qué debe hacer una persona" (p. 225).

Es necesario diferenciar entre el razonamiento moral orientado a la justicia, es decir el razonamiento sobre dilemas morales en los que las normas y leyes u obligaciones formales son centrales (Kohlberg, 1981), del razonamiento relacionado con una moralidad positiva como el razonamiento moral prosocial (Eisenberg, 1986). Este último se refiere a dilemas sociales en los que las necesidades o deseos de una persona están en conflicto con las necesidades o deseos de otra u otras personas, en un contexto donde el papel de las leyes, normas, dictados de las autoridades, prohibiciones y castigos, es mínimo (Carlo, Einsenberg, Koller, Da Silva \& Frohlich, 1996; Eisenberg, Lennon \& Roth, 1983; Eisenberg, Miller, Shell, McNalley \& Shea, 1991).
Las distintas categorías de razonamiento utilizadas por los niños fueron agrupadas en diferentes niveles de razonamiento moral prosocial, donde la progresión de un estadio al siguiente viene precedida por el uso incipiente de un nivel más alto de razonamiento (Eisenberg et al., 1983). Los cambios en el razonamiento moral prosocial relacionados con la edad que se han encontrado en la infancia y adolescencia temprana, generalmente han sido consistentes con el punto de vista de Kohlberg (1981) acerca de que las capacidades de toma de perspectiva compleja y de comprensión de conceptos abstractos, están asociadas con el avance del razonamiento moral.

Eisenberg (1986) encontró que los niños pequeños tienden a usar principalmente el razonamiento hedonista u orientado a las necesidades (empático primitivo) y que, durante la escuela primaria, el razonamiento comienza a reflejar preocupación por la aprobación e intensificación de las relaciones interpersonales, así como el deseo de comportarse de una forma estereotipadamente aceptable, aunque este razonamiento va disminuyendo en la niñez tardía (finales de la escuela primaria). El razonamiento de reciprocidad directa, que refleja una orientación hacia el autobeneficio, aumenta en la escuela primaria (Eisenberg et al., 1987).

Eisenberg et al. (1991) encuentran que en los adolescentes conviven tipos de razonamiento moral prosocial cognitivamente avanzados con otros menos evolucionados, no obstante lo cual, afirman que hay una progresiva sofisticación cognitiva desde el razonamiento moral prosocial hedonista al orientado a la aprobación, al orientado a las necesidades, al estereotipado y al internalizado.

Dado que aparentemente determinados componentes del razonamiento moral prosocial se relacionan positivamente con la conducta altruista prosocial (Carlo et al., 1996; Eisenberg, 1991; Eisenberg, Carlo, Murphy \& Van Court, 1995; Mestre, Pérez-Delgado, Samper \& Fuentes, 1999) y que es posible intervenir sobre el mismo (Eisenberg et al., 1983), es de máxima importancia poder evaluar este razonamiento con instrumentos objetivos que faciliten un diagnóstico válido y confiable. 
Carlo, Eisenberg y Knight (1992) desarrollaron una medida objetiva de razonamiento moral prosocial (PROM por sus siglas en inglés) con la que se superan las limitaciones de las entrevistas de razonamiento moral y se facilita la valoración del razonamiento moral de los adolescentes. Sin embargo, la dificultad de desarrollar un instrumento similar para niños pequeños, radica en que éstos no tienen un lenguaje tan evolucionado y les cuesta sostener la atención, tanto para retener todas las alternativas de respuesta y ordenarlas jerárquicamente, como para responder a un número de historias mayor a cuatro (Richaud, Lemos \& Schulz, 2007).

El objetivo del presente trabajo fue desarrollar una prueba objetiva pictórica de razonamiento prosocial, para niños de 7 y 8 años, basada en una anterior de tipo verbal, y estudiar en forma preliminar su funcionamiento psicométrico.

A continuación se presentan los niveles de razonamiento moral prosocial que se consideran posibles de evaluar en niños de 7 y 8 años, teniendo en cuenta el desarrollo cognitivo de ese período evolutivo. La siguiente taxonomía toma conceptos desarrollados por Einsenberg, Lennon y Roth (Eisenberg, 1979; Eisenberg et al., 1983, 1987, 1991, 1995).

\section{Estudio 1: Versión verbal del instrumento}

Con base en la versión española del Prosocial Reasoning Objective Measurement (PROM), adaptada y validada por Mestre, Frías, Samper y Tur (2002), se elaboraron seis historias que presentaban distintas situaciones conflictivas en las que los niños debían identificarse con los personajes principales y sugerir cómo resolver un dilema social. Se crearon dos versiones paralelas de cada historia, una para varones y otra para nenas (Richaud, Lemos \& Schulz, 2007).

Las historias presentan al niño situaciones determinadas en las que están implicadas conductas de compartir y ayudar, material o emocionalmente, teniendo en cuenta los estudios de Eisenberg, Murphy, Shepard, Cumberland y Carlo (1999),

\section{TABLA 1}

Niveles de razonamiento moral prosocial considerados factibles de evaluar en niños de 7 y 8 años

\begin{tabular}{|c|c|}
\hline Orientación & Descripción \\
\hline Hedonista & $\begin{array}{l}\text { El niño está interesado por las conse- } \\
\text { cuencias para sí mismo más que por } \\
\text { consideraciones morales. Las consi- } \\
\text { deraciones para ayudar o no ayudar } \\
\text { al otro incluyen la consideración del } \\
\text { beneficio directo para uno mismo, } \\
\text { futura reciprocidad e interés por otros } \\
\text { a quienes el individuo necesita y/o } \\
\text { aprecia (debido al lazo afectivo). Ej.: } \\
\text { le convidé una galletita porque así ella me } \\
\text { convida mañana. }\end{array}$ \\
\hline
\end{tabular}

Orientado El niño expresa interés por las necesia las necesi- dades físicas, materiales y psicológicas dades de los de otros aun cuando éstas confluyen otros con las propias. Este interés es expresado en los términos más simples, a falta de clara evidencia de toma de perspectiva autorreflexiva, expresiones verbales de simpatía o referencia a afecto internalizado tal como la culpa. Ej.: le convidé una galletita porque ella tenía hambre.

Centrado en Las consideraciones de aprobación y la aproba- aceptación de otros son usadas en la ción de los justificación de conductas prosociales o demás. no prosociales. Ej.: le convidé una galletita porque sé que a la seño le va a gustar que haga eso.

Estereoti- Las imágenes estereotipadas de buenas pado o malas personas y/o conductas son utilizadas en la justificación de la conducta prosocial o no prosocial. Ej.: le convidé una galletita porque es bueno compartir.

Orientación Los juicios del niño incluyen la eviempática dencia de reacción simpática, ex(simpática) presión de preocupación simpática y cuidado hacia otros. Ej.: le convidé una galletita porque me dio lástima que no haya traído merienda.

Afecto in- El niño piensa en las consecuencias ternalizado afectivas que producirá su accionar. primitivo Ej.: le convidé una galletita para que ella se sienta mejor.

Fuente: elaboración propia con base en Eisenberg et al. (1983). 
acerca de los comportamientos a través de los cuales los niños pequeños manifiestan el razonamiento prosocial.

Cada historia está estructurada de la siguiente manera: en primer lugar, se presenta la situación y luego una pregunta destinada a evaluar qué debería hacer el personaje principal en tal situación. Luego, se le pregunta al niño por qué el protagonista debería actuar de esa forma, incentivándolo a que dé dos o tres respuestas espontáneas.

A continuación se clasifican las respuestas dadas por el niño dentro de las opciones que aparecen en el instrumento, y luego, en esta primera versión verbal, se le leen, en orden contrabalanceado, el resto de las posibles opciones de resolución de la situación no expresadas de manera espontánea, pidiéndole que las ordene de acuerdo a su importancia.

A continuación se presenta un ejemplo de una de las historias de la primera versión verbal con sus correspondientes opciones de respuesta.

Historia: María había llevado a la escuela unas galletitas para la merienda. Su mamá le había comprado un paquete chico de las galletitas que más le gustaban. Las galletitas eran riquísimas, el único problema es que eran muy poquitas. Julia, una compañerita de María, que no había llevado merienda, se acercó a ella en el recreo.

Preguntas:

1. ¿Qué tendría que hacer María?

2. ¿Por qué tendría que hacer eso?

Opciones de respuesta:

a. Porque Julia tenía hambre (orientado a la necesidad).

b. Porque así otro día Julia le convidará a ella (hedonista).

c. Porque es bueno compartir (estereotipado).

d. Porque su mamá se va a poner contenta por lo que hizo (búsqueda de aprobación).

e. Porque le dio lástima que nadie le comparta a Julia (orientación empática).

f. Para que Julia se sienta bien (afecto internalizado primitivo).
Los resultados obtenidos en esta primera versión verbal del instrumento (Richaud, Lemos \& Schulz, 2007) indicaron que:

1. Resulta dificultoso construir historias breves, claras, donde se presente un dilema y donde las alternativas de respuesta que se ofrezcan sean pocas, ya que al niño se le dificulta mucho tener que recordar seis alternativas verbales simultáneas para poder jerarquizarlas. Cuando los niños daban menos de tres respuestas espontáneas, el número de alternativas que se les debía presentar era mayor, siendo más difícil su jerarquización. En estos casos, en general los niños escogían la última opción. Este sesgo se trató de controlar presentando las alternativas de manera balanceada, es decir alternando el orden de presentación.

2. El contenido de las historias influyó sobre las respuestas de los niños.

3. Cuando se estudió la consistencia interna del instrumento, los alpha de Cronbach oscilaron entre .28 y .53 . Si bien los alpha fueron bajos, es sabido que las respuestas de los niños son más inestables que las de los adultos. Estos resultados también pudieron haber sido afectados por el tamaño de la muestra, por el sistema de puntuación establecido -basado en el orden de elección de las respuestas-, y por el orden aleatorio de presentación de las alternativas de respuesta, con lo que creaba mayor fluctuación en los valores asignados a cada razonamiento que si se hubiera trabajado con una escala Lickert, cosa que era imposible con niños tan pequeños.

4. También se encontró una influencia de la desiderabilidad social sobre las respuestas, lo que por otra parte era esperable en niños de esta edad (Lemos, 2006), y que además indica una percepción de parte de los niños de lo que se espera socialmente, o una forma primitiva de estereotipo social.

5. Con respecto a la validez de constructo, se encontró, en primer lugar, que el patrón de diferencias por sexo en el razonamiento prosocial, tal como ha sido evaluado por esta prueba, sigue las pautas establecidas por otros investigadores 
(Carlo et al., 1996; Mestre, Pérez-Delgado \& Escrivá, 1995). También se obtuvieron resultados con respecto a la edad coincidentes con lo afirmado por Eisenberg et al. (1995). Por último, al analizar las correlaciones entre los diferentes tipos de razonamiento prosocial, se encontró un patrón en línea con el modelo teórico.

\section{Estudio 2: Segunda versión del instrumento}

En función de los resultados obtenidos y las dificultades encontradas en la primera versión verbal del instrumento detalladas anteriormente, se desarrolló una segunda versión pictórica en la cual además,

1. Se ajustaron los relatos procurando mayor neutralidad.

2. La pregunta: "iPor qué tendría que hacer eso?" se cambió por: "¿En qué pensó para hacer eso?”, con los objetivos de: a) poder captar mejor el proceso de pensamiento que lleva al niño a la decisión de la respuesta dada; b) mantener una modalidad más semejante a la versión original del PROM; y, c) disminuir la posible inducción de la respuesta (ej.: "su mamá se va a poner contenta por lo que hizo", por "iqué pensará mi mamá?")

3. Se reagruparon las categorías de razonamiento prosocial que implican la consideración por el otro (orientado a la necesidad, afecto internalizado primitivo y orientación empática), reduciendo de este modo a cuatro los razonamientos evaluados.

4. Se diseñó una nueva forma de presentación pictórica de las alternativas, para evitar los problemas de memoria, mostrando a los niños todas las opciones de respuesta restantes a las espontáneas, de manera simultánea, para que las ordenara.

En la Figura 1, se presentan las alternativas de respuesta pictóricas para la misma historia presentada anteriormente.

FIGURA 1

Alternativas de respuesta pictóricas para la primera historia
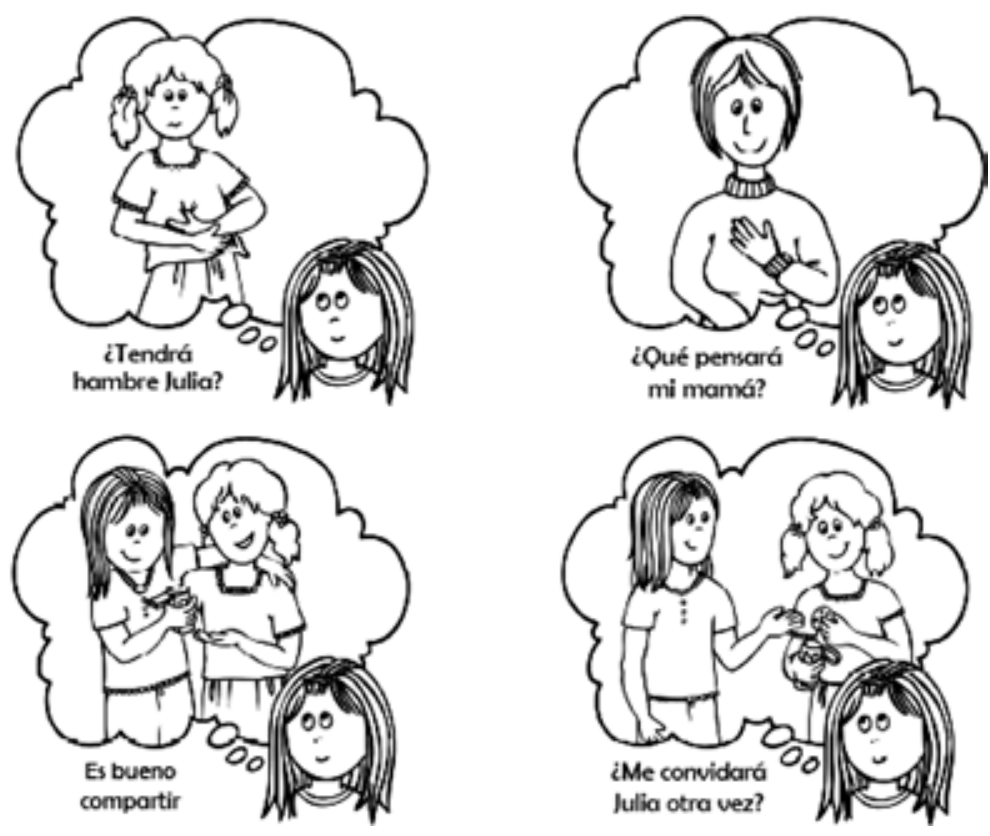

Fuente: elaboración propia. 


\section{Método}

\section{Participantes}

En este segundo estudio, participaron 125 niños de 7 y 8 años de edad, de nivel socioeconómico medio, asistentes a las escuelas La Salle y Rivadavia, privada confesional la primera y pública la segunda, ambas de la ciudad de Paraná, Entre Ríos. En todos los casos, se solicitó previamente el consentimiento informado de los padres.

\section{Procedimientos para el análisis de datos}

1. Se evaluó la consistencia interna de la escala a través del coeficiente alpha de Cronbach, estimando la homogeneidad para cada tipo de razonamiento moral prosocial.

2. Con el objetivo de comparar los tipos de razonamiento prosocial predominantes por sexo y por edad, se realizaron diferencias de medias a través de diferentes pruebas $t$ para muestras independientes y gráficos de perfiles.

3. Con el objetivo de evaluar la relación entre los diferentes tipos de razonamiento, se realizaron análisis de correlaciones de Pearson.

4. Para evaluar si el contenido de las historias sesgaba el tipo de razonamiento utilizado, se calcularon las frecuencias de razonamiento por historia.

5. Con el fin de determinar cuáles son los tipos de razonamiento prosocial predominantes a los $7 \mathrm{y}$ 8 años, se calcularon las frecuencias utilizadas para cada tipo de razonamiento.

\section{Resultados}

\section{Análisis de la consistencia interna}

En las Tablas 2 y 3 se presentan, respectivamente, la comparación de los coeficientes alpha correspondientes a la $1^{\mathrm{O}}$ y $2^{\underline{o}}$ versión y los índices de consistencia interna según la edad.
TABLA 2

Comparación de la consistencia interna (alpha de Cronbach) entre la $1^{\mathrm{o}}$ y $2^{\mathrm{o}}$ versión del instrumento

\begin{tabular}{lcc}
\hline Tipo de razonamiento & $\alpha$ 1ㅇv versión & $\alpha$ 2º versión \\
\hline Orientado Necesidad & 0.39 & 0.55 \\
Hedonista & 0.53 & 0.56 \\
Estereotipado & 0.28 & 0.53 \\
Centrado Aprobación & 0.43 & 0.50 \\
\hline
\end{tabular}

Fuente: elaboración propia.

TABLA 3

Comparación de la consistencia interna (alpha de Cronbach)entre niños de 7 y 8 años

\begin{tabular}{lccc}
\hline Tipo de razonamiento & $\alpha$ general & $\alpha$ 7 años & A 8 años \\
\hline Orientado Necesidad & 0.55 & 0.59 & 0.49 \\
Hedonista & 0.56 & 0.53 & 0.58 \\
Estereotipado & 0.53 & 0.38 & 0.62 \\
Centrado Aprobación & 0.50 & 0.48 & 0.52 \\
\hline
\end{tabular}

Fuente: elaboración propia.

Como se puede observar, la consistencia interna mejoró en la $2^{\mathrm{o}}$ versión del instrumento en todos los tipos de razonamientos evaluados, manteniéndose también más semejantes entre sí.

En cuanto a las variaciones de los coeficientes alpha en función de la edad, como es de esperar, el índice más bajo se presentó a los 7 años, y precisamente en el razonamiento estereotipado. Según Eisenberg (1995) es un tipo de razonamiento menos desarrollado en los niños pequeños y que recién se consolida entre los 9 y 12 años, para luego comenzar a disminuir.

\section{Comparación de los tipos de razonamiento según sexo}

Como se puede observar en la Tabla 4, las niñas otorgaron mayor jerarquía, en comparación con los varones, a los razonamientos estereotipado y orientado a la necesidad, mientras que los varones, por 
su parte, privilegiaron los razonamientos hedonista y centrado en la aprobación del otro.

\section{TABLA 4}

Comparación de las medias de los tipos de Razonamiento Prosocial según sexo

\begin{tabular}{|c|c|c|c|c|c|c|}
\hline \multirow{2}{*}{ Razonamiento } & \multicolumn{2}{|c|}{ Mujer } & \multicolumn{2}{|c|}{ Varón } & \multirow[t]{2}{*}{$t$} & \multirow[t]{2}{*}{$p$} \\
\hline & M & $\mathrm{DE}$ & $\mathbf{M}$ & $\mathrm{DE}$ & & \\
\hline Estereotipado & 12.88 & 2.09 & 11.61 & 2.54 & 2.95 & 0.004 \\
\hline $\begin{array}{l}\text { Orientado } \\
\text { Necesidad }\end{array}$ & 12.46 & 2.52 & 11.37 & 3.10 & 2.12 & 0.036 \\
\hline Hedonista & 8.44 & 1.98 & 9.20 & 2.95 & 1.63 & 0.099 \\
\hline $\begin{array}{l}\text { Centrado } \\
\text { Aprobación }\end{array}$ & 6.23 & 2.02 & 7.76 & 2.27 & 3.91 & 0.000 \\
\hline
\end{tabular}

Fuente: elaboración propia.

\section{Diferencias en los tipos de razonamiento según edad}

En cuanto a las diferencias en los tipos de razonamiento moral prosocial según la edad, tal como era de esperar en concordancia con la teoría, los niños de 7 años privilegiaron los razonamientos hedonista y centrado en la aprobación, y los de 8 años, los razonamientos orientados a la necesidad y estereotipado (véase Tabla 5).

\section{TABLA 5}

Comparación entre las medias de tipos de Razonamiento Prosocial por edad

\begin{tabular}{lcccccc}
\hline \multirow{2}{*}{ Razonamiento } & \multicolumn{2}{c}{7 años } & \multicolumn{2}{c}{8 años } & $t$ & $p$ \\
\cline { 2 - 8 } & $\mathrm{M}$ & $\mathrm{DE}$ & $\mathrm{M}$ & $\mathrm{DE}$ & & \\
Orientado & 11.88 & 3.15 & 12.02 & 2.46 & 2.70 & 0.794 \\
Necesidad & & & & & & \\
Estereotipado & 11.67 & 2.24 & 13.06 & 2.37 & 3.28 & 0.001 \\
Hedonista & 9.33 & 2.49 & 8.10 & 2.35 & 2.72 & 0.007 \\
$\begin{array}{l}\text { Centrado } \\
\text { Aprobación }\end{array}$ & 7.12 & 2.26 & 6.73 & 2.24 & .93 & 0.347 \\
\hline
\end{tabular}

Fuente: elaboración propia.

\section{Relación entre los tipos de razonamiento}

Como se observa en la Tabla 6, se presentaron correlaciones negativas y significativas entre los distintos tipos de razonamientos prosociales, con excepción de la relación entre el razonamiento estereotipado y hedonista.

\section{TABLA 6}

Correlación entre de los tipos de Razonamiento

Prosocial

\begin{tabular}{lccc}
\hline & $\begin{array}{r}\text { Orientado } \\
\text { Necesidad }\end{array}$ & Hedonista & Estereotipado \\
\hline Hedonista & $-0.579(* *)$ & & \\
Estereotipado & $-0.370(* *)$ & -0.135 & \\
Centrado & $-0.210(*)$ & $-.0226(*)$ & $-0.433(* *)$ \\
Aprobación & & & \\
\hline
\end{tabular}

** La correlación es significativa al nivel 0.01 (bilateral). * La correlación es significativa al nivel 0.05 (bilateral).

Fuente: elaboración propia.

\section{Comparación de los tipos de razonamiento por historia}

Para evaluar si el contenido de las historias sesgaba el tipo de razonamiento utilizado, se calcularon las medias según el tipo de razonamiento por historia.

Como se puede observar en las siguientes figuras (véanse Figuras 2 a 5), la jerarquía en los tipos de razonamiento escogidos en cada historia, se mantuvo de manera consistente. Obsérvese en la Figura 6 la variabilidad que se presentaba entre cada una de las historias, en la primera versión del instrumento. 
FIGURA 2

Comparación de los tipos de los tipos de razonamiento en la historia 1

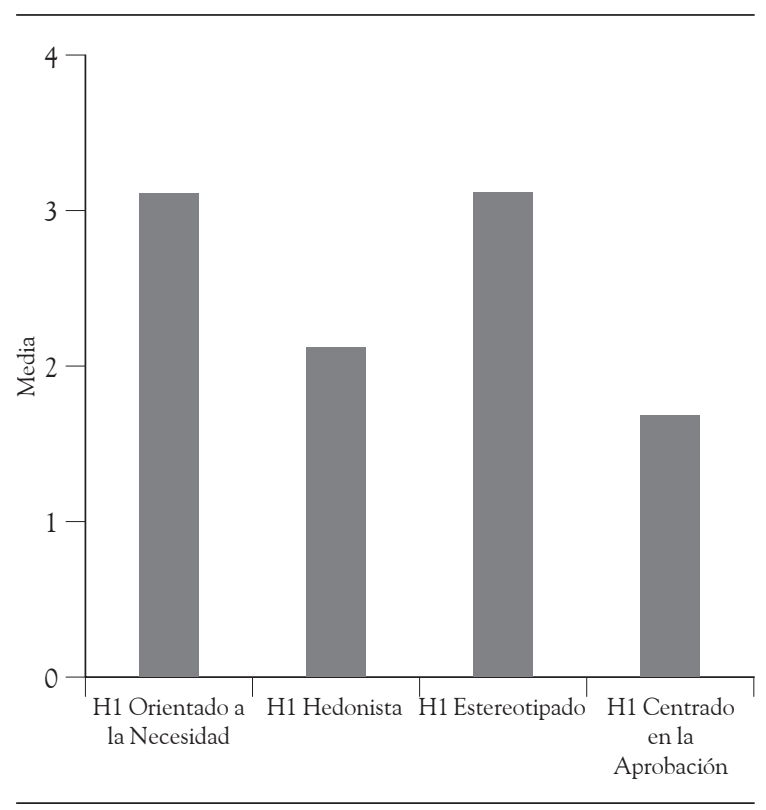

Fuente: elaboración propia.

FIGURA 3

Comparación de los tipos de razonamiento en la historia 2

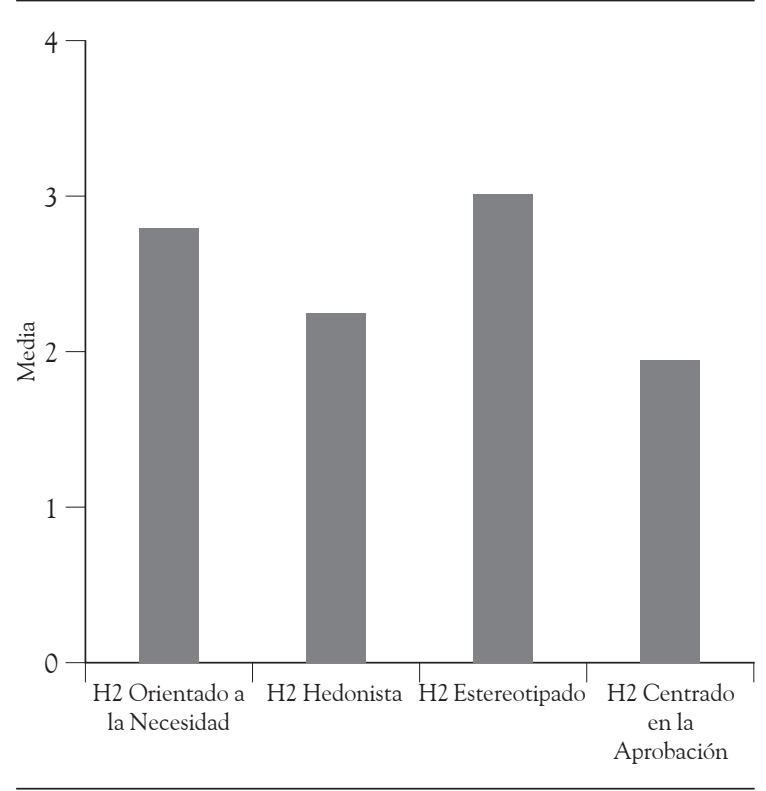

Fuente: elaboración propia.

\section{Figura 4}

Comparación de tipos de razonamiento en la historia 3

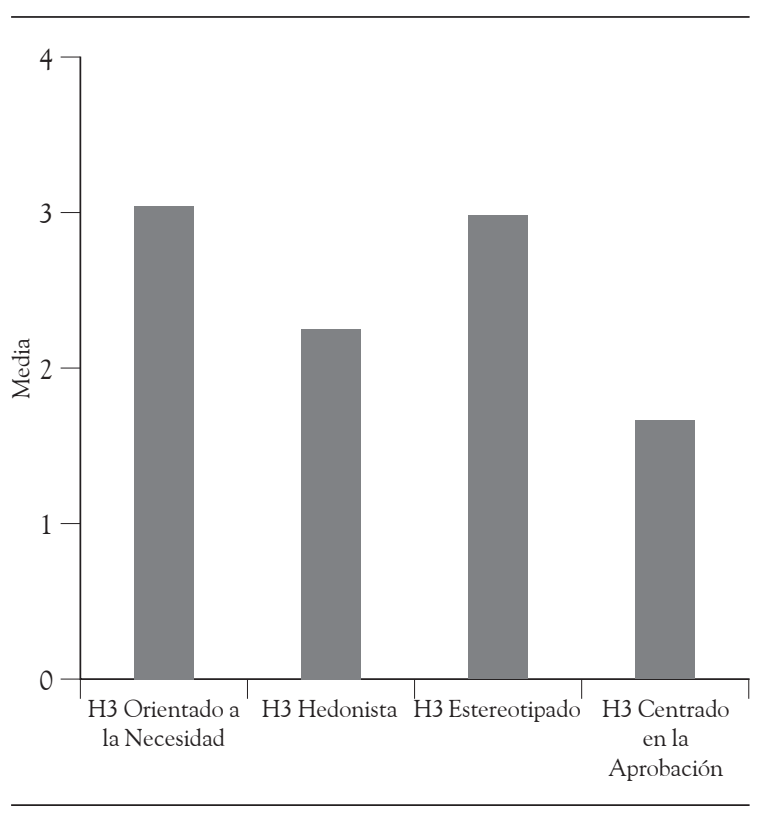

Fuente: elaboración propia.

\section{FiguRA 5}

Comparación de tipos de razonamiento en la historia 4

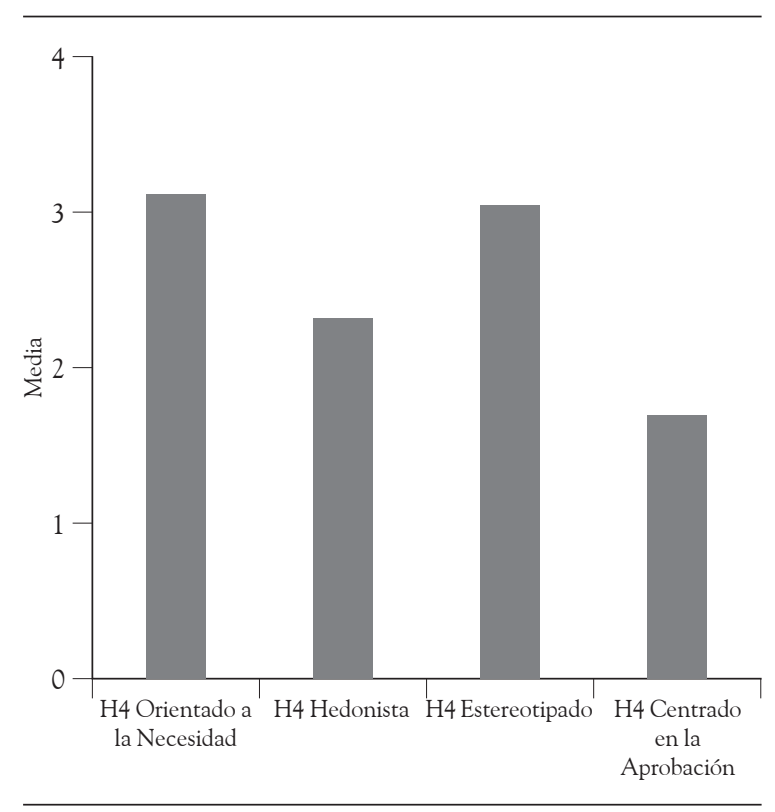

Fuente: elaboración propia. 


\section{Figura 6}

Comparación entre la $1^{\underline{o}}$ y $2^{\underline{o}}$ versión de la consistencia de las frecuencias de los tipos de razonamiento jerarquizados por historia

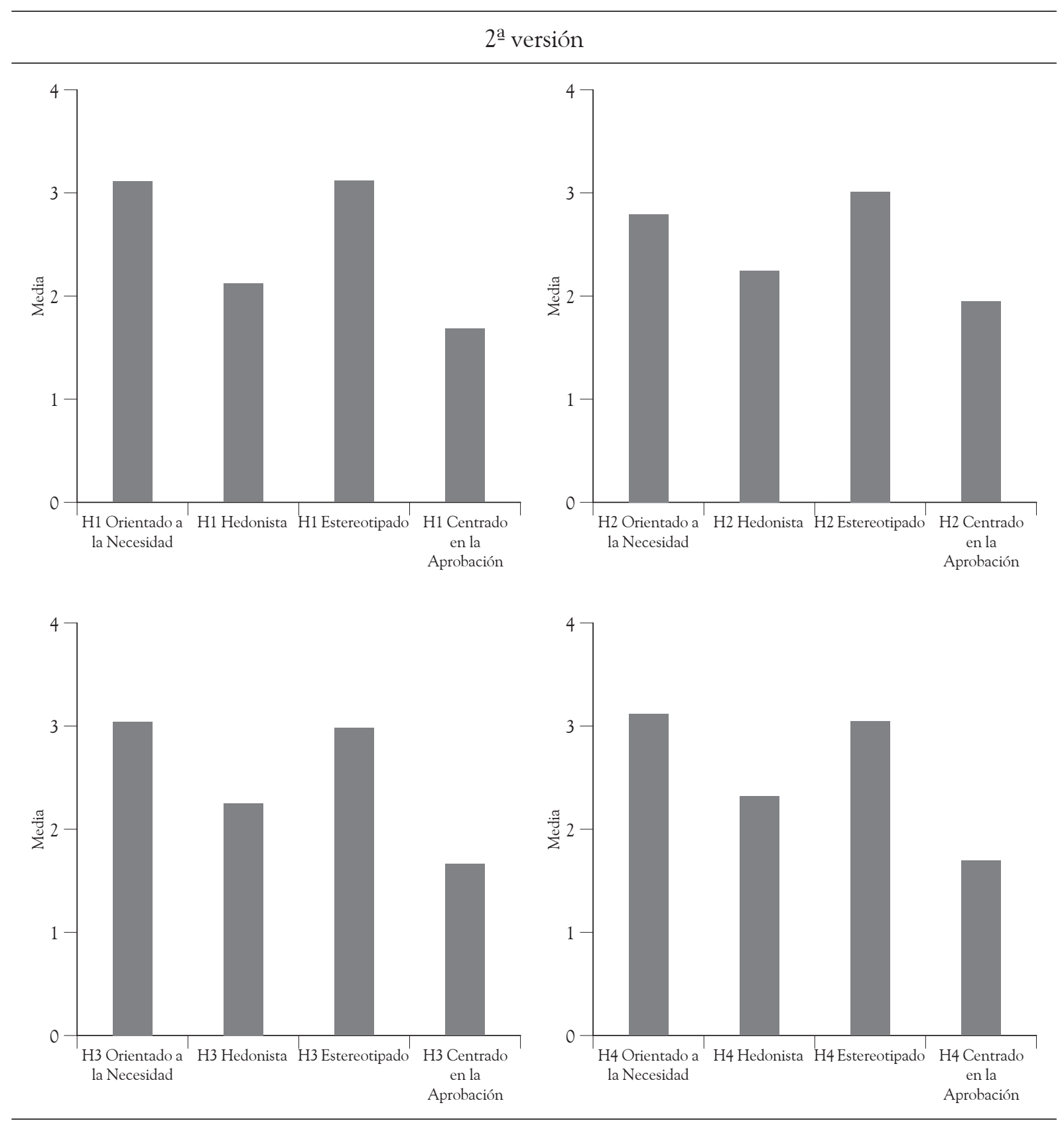




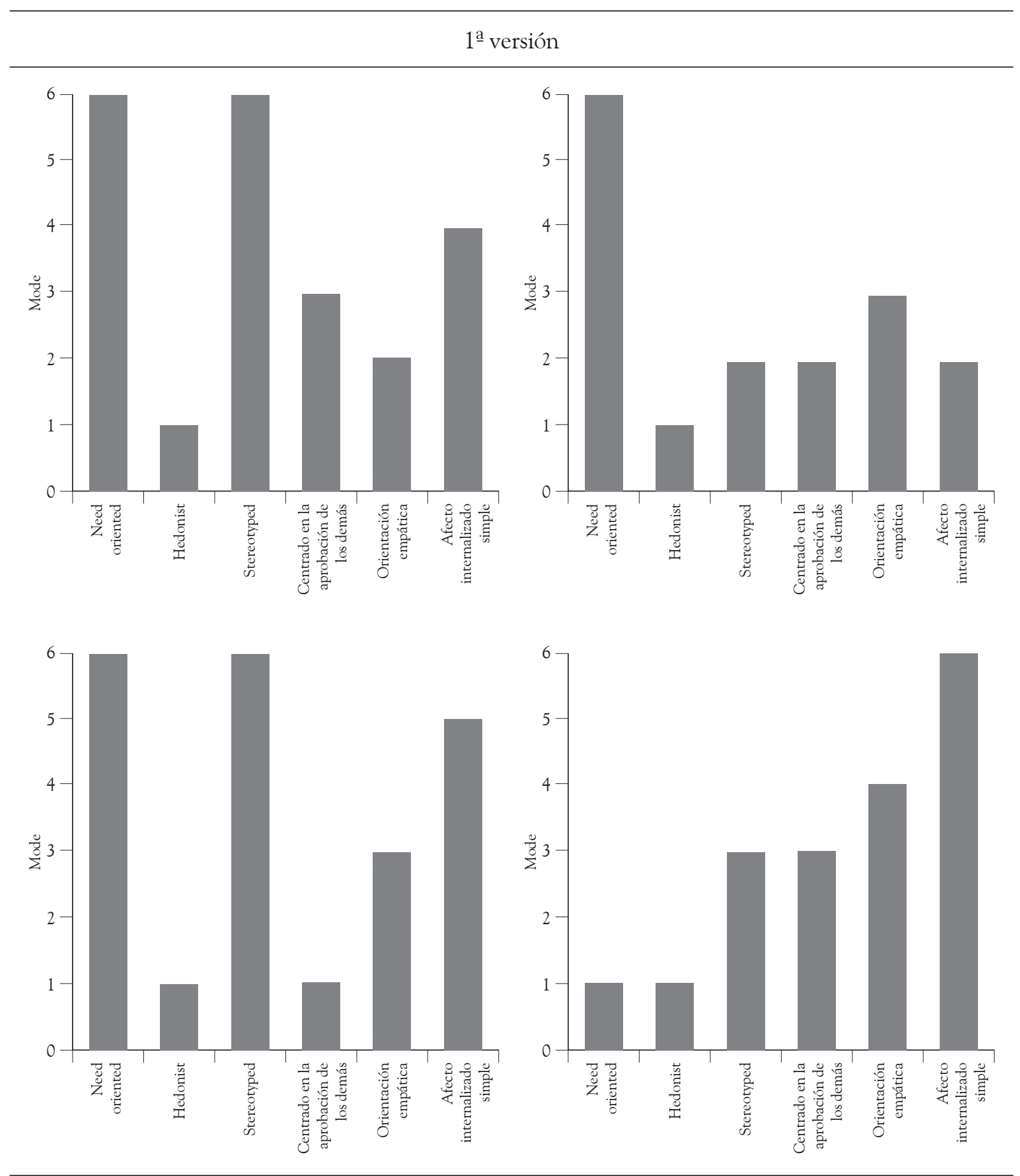

Fuente: elaboración propia. 


\section{Jerarquización de los tipos de razonamiento utilizados entre los 7 y 8 años}

Con el fin de determinar cuáles son los tipos de razonamiento prosocial predominantes a los $7 \mathrm{y}$ 8 años, se calcularon las medias para cada tipo de razonamiento. Como se observa en la Tabla 7, la justificación de la conducta prosocial viene dada por los siguientes razonamientos prosociales, que en orden de importancia son: estereotipado, orientado a la necesidad, hedonista y centrado en la aprobación.

\section{TABLA 7}

Comparación de medias de los tipos de razonamiento prosocial utilizados a los 7 y 8 años

\begin{tabular}{lcc}
\hline \multicolumn{1}{c}{ Razonamiento } & $\mathrm{M}$ & $\mathrm{DE}$ \\
\hline Estereotipado & 12.28 & 2.39 \\
Orientado Necesidad & 11.94 & 2.85 \\
Hedonista & 8.79 & 2.50 \\
Centrado Aprobación & 6.95 & 2.24 \\
\hline
\end{tabular}

Fuente: elaboración propia.

\section{Discusión}

Los niños, a menudo, se enfrentan con la decisión de ayudar a otros y esta decisión implica algún costo para sí mismos. Muchas veces, estas decisiones son difíciles ya que deben ser tomadas en un contexto en que los lineamientos externos están ausentes o no son claros. Teniendo en cuenta, como se mencionó en un principio, que el razonamiento moral prosocial mantiene una relación positiva con la conducta altruista prosocial (Carlo et al., 1996; Eisenberg, 1991, 1995; Mestre et al., 1999), y que es posible intervenir sobre el razonamiento prosocial (Eisenberg, Lennon \& Roth, 1983) es fundamental desarrollar una medida que permita evaluar este constructo de manera válida y confiable.

En este trabajo hemos analizado una versión pictórica de un instrumento para evaluar el razonamiento prosocial en niños de 7 y 8 años.

\section{Consistencia interna}

Si bien los coeficientes alpha son algo bajos, al compararlos con los de la primera versión todos mejoraron, pudiéndose considerar aceptables, dado que, por una parte, se trata de niños pequeños que son conocidamente inestables y, por otra, que se trata de un constructo sumamente difícil de evaluar en niños de esta edad.

\section{Diferencias según el sexo}

Las niñas, que presentaron diferencias significativas en comparación con los niños, jerarquizaron más los razonamientos estereotipado y orientado hacia las necesidades del otro. Los niños, por su parte, otorgaron mayor jerarquía al razonamiento hedonista y centrado en la aprobación del otro.

Las diferencias encontradas por sexo, en el razonamiento prosocial, siguen las pautas establecidas por otros investigadores, aunque trabajando con adolescentes (Carlo et al., 1996; Mestre et al., 1995). Así, las niñas tendieron a superar a los varones en razonamiento estereotipado y orientado hacia las necesidades del otro, y los varones tendieron a superar a las niñas en hedonismo y centrado en la aprobación.

\section{Diferencias según la edad}

Es posible notar que los niños de 8 años adjudicaron mayor importancia a los razonamientos estereotipado y orientado a la necesidad del otro, mientras que los de 7 años privilegiaron los razonamientos hedonista y centrado en la aprobación. Estos resultados también coinciden con lo ya mencionado por Eisenberg et al. (1995).

\section{Relación entre los diferentes Tipos de Razonamiento Prosocial}

A pesar de las dificultades encontradas en relación con la consistencia interna, al tratar de analizar si este instrumento presenta validez de constructo, se encontraron correlaciones entre los diferentes tipos de razonamiento prosocial, en línea con la 
teoría: el hedonismo tiene correlaciones negativas con el resto de los razonamientos, con el centrado en la necesidad, con el estereotipado o el deber ser y, en menor medida pero también significativo, con el centrado en la aprobación.

El razonamiento orientado a la necesidad de los otros correlaciona negativamente con el hedonista, el estereotipado y con el centrado en la aprobación de los otros, como si la necesidad del otro predominara sobre lo impuesto externamente.

El razonamiento estereotipado se relaciona negativamente con el orientado a la necesidad, con la búsqueda de aprobación y con el hedonista.

Estos resultados mantuvieron un patrón en línea con el modelo teórico, en tanto el hedonismo se correlacionó negativamente con el resto de los razonamientos más dependientes u orientados hacia el otro; el razonamiento centrado en la necesidad de los otros se correlacionó negativamente con los razonamientos más orientados externamente, estereotipado y centrado en la aprobación de los otros; mientras que éstos últimos también correlacionaron negativamente con orientado a la necesidad del otro.

\section{Consistencia entre los tipos de razonamientos escogidos por historia}

Como pudo observarse en los resultados en relación a este aspecto, la jerarquización de los diferentes razonamientos se mantuvo consistente en las diferentes historias. Los razonamientos más jerarquizados, en todas las historias, fueron el estereotipado y el orientado a la necesidad, y los menos, fueron el hedonista y el centrado en la búsqueda de aprobación. Esta consistencia apoya la neutralidad del contenido de las historias, ya que el tipo de razonamiento privilegiado no varió en función de las mismas.

\section{Predominio general de los tipos de razonamientos prosociales utilizados entre los 7 y 8 años}

En general, en las historias y en el promedio total, puede observarse que se privilegió, en primer lugar, el razonamiento estereotipado; en segundo lugar, el orientado a la necesidad del otro; en tercer lugar el hedonista y, en cuarto lugar, el centrado en la aprobación.

Según Eisenberg (1986), durante la escuela primaria, comienzan a intensificarse las relaciones interpersonales, así como el deseo de comportarse de forma estereotipadamente aceptable, aunque este razonamiento va disminuyendo en la niñez tardía (finales de la escuela primaria).

A estas edades se espera que el hedonismo vaya disminuyendo y que las otras formas de razonamiento se mantengan o aumenten paulatinamente. Así también, se espera que el razonamiento centrado en la aprobación del otro, típico del niño preescolar, vaya disminuyendo (Eisenberg et al., 1995).

Determinados razonamientos prosociales se relacionan positivamente con la conducta prosocial altruista. Los comportamientos prosociales son considerados hoy como la mejor estrategia para prevenir y afrontar la creciente expresión de la agresividad y la violencia infantil. Pero no sólo esto, constituyen también, para numerosos autores, un verdadero factor protector y optimizador de la salud mental en general (Palau del Pulgar, 2006; Roche, 1998).

Estudiar y fortalecer este importante recurso psicológico, es un aporte importante para la psicología positiva. Este trabajo representa un avance en este sentido, presentando una escala para evaluar el razonamiento moral prosocial en niños de entre 7 y 8 años de edad, con probados criterios de validez de constructo y aceptables índices de confiabilidad.

\section{Referencias}

Carlo, G., Eisenberg, N. \& Knight, G. P. (1992). An objective measure of adolescents prosocial moral reasoning. Journal of Research on Adolescence, 2, 331-349.

Carlo, G., Einsenberg, N., Koller, S., Da Silva, M. \& Frohlich, C. (1996). A cross-national study on the relations among prosocial moral reasoning, gender role orientations, and prosocial behaviors. Developmental Psychology, 32, 234-240. 
Eisenberg, N. (1979). Development of children's prosocial moral judgment. Developmental Psychology, $15,128-137$.

Eisenberg, N. (1986). Altruistic emotion, cognition and behavior. Hillsdale, NJ: Erlbaum.

Eisenberg, N. (1991). Values, sympathy, and individual differences: Toward pluralism of factor influencing altruism and empathy. Psychological Inquiry, 2 (2), 128-131.

Eisenberg, N., Carlo, G., Murphy, B. \& Van Court, P. (1995). Prosocial development in late adolescence: A longitudinal study. Child Development, 66, 1179-1197.

Eisenberg, N., Lennon, R. \& Roth, K. (1983). Prosocial development in childhood: A longitudinal study. Developmental Psychology, 19, 846-855.

Eisenberg, N., Miller, P., Shell, R., McNalley, S. \& Shea, C. (1991). Prosocial development in adolescence: A longitudinal study. Developmental Psychology, 27, 849-857.

Eisenberg, N., Murphy, B., Shepard, S., Cumberland, A. \& Carlo, G. (1999). Consistency and development of prosocial dispositions: A longitudinal study. Child Development, 70 (6), 1360-1372.

Eisenberg, N. \& Roth, K. (1980). Development of young childrens's prosocial moral judgment: A longitudinal follow-up. Developmental Psychology, 16, 375-376.

Eisenberg, N., Shell, R., Pasternack, J., Lennon, R., Beller, R. \& Mathy, R. (1987). Prosocial development in middle childhood: A longitudinal study. Developmental Psychology, 23, 712-718.

Kohlberg, L. (1981). Essays on moral development: The philosophy of moral development: Moral stages and the idea of justice (Vol. 1). San Francisco: Harper $\&$ Row.

Lemos, V. (2006). La deseabilidad social en la evaluación de la personalidad infantil. Suma Psicológica, $13(1), 7-14$.
López, F. (1994). La conducta altruista. Teoría, investigación e intervención educativa. Estrella: EVD.

Mestre, V., Frías, D., Samper, P. \& Tur, A. (2002). Adaptación y validación en población Española del PROM: una medida objetiva del razonamiento moral prosocial. Acción Psicológica, 3, 221-232.

Mestre, V., Pérez-Delgado, E. \& Escrivá, A. (1995). Lo cognitivo vs. lo afectivo. Desarrollo moral y personalidad. En E. Pérez-Delgado \& M. V. Mestre (Coords.), Psicología moral y crecimiento personal (pp. 155-179). Barcelona: Ariel.

Mestre, V., Pérez-Delgado, E., Samper, P. \& Fuentes, E. (1999). Razonamiento moral prosocial. Descripción y teoría. En E. Pérez-Delgado \& M. V. Mestre (Coords.), Psicología moral y crecimiento personal (pp. 243-258). Barcelona: Ariel.

Palau del Pulgar, M. (2006, abril). La educación prosocial aportación al aprendizaje servicio. Ponencia presentada en el Seminario Internacional de Aprendizaje Servicio. Una herramienta para el servicio comunitario, Caracas, Venezuela.

Pérez-Delgado, E. (1995). Psicología, ética, religión. iÉtica versus religión? Madrid: Siglo XXI.

Richaud de Minzi, M. C., Lemos, V. \& Schulz, A. (2007, junio). Evaluación del razonamiento prosocial en niños de 7 y 8 años. En M. C. Richaud de Minzi (Presidente), Evaluación de las competencias sociales en la infancia y adolescencia. Simposio efectuado en la reunión del VI Congreso Iberoamericano de Evaluación Psicológica, Ciudad de México, México.

Roche Olivar, R. (1998). Educación prosocial de las emociones, valores y actitudes positivas: para adolescentes en entornos familiares y escolares. Barcelona: Blume.

Turiel, E. (1983). El conocimiento social, moralidad y convención. Debate, 12, 28-50. 
